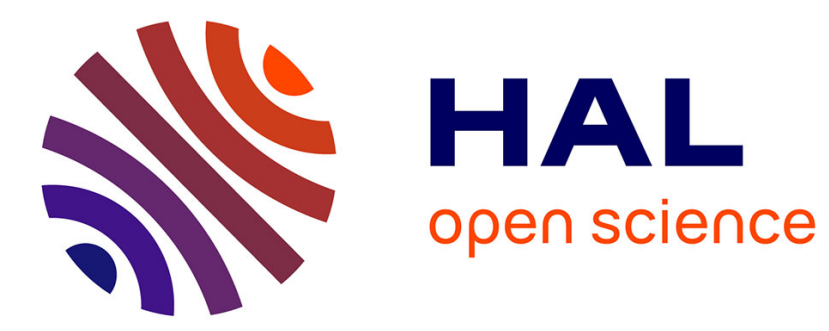

\title{
Adaptation of the Simple or Complex nature of V1 receptive fields to visual statistics
}

Julien Fournier, Cyril Monier, Marc Pananceau, Yves Fregnac

\section{To cite this version:}

Julien Fournier, Cyril Monier, Marc Pananceau, Yves Fregnac. Adaptation of the Simple or Complex nature of V1 receptive fields to visual statistics. Nature Neuroscience, 2011, 14 (8), pp.1053-60. 10.1038/nn.2861. hal-00660536

\section{HAL Id: hal-00660536 https://hal.science/hal-00660536}

Submitted on 17 Jan 2012

HAL is a multi-disciplinary open access archive for the deposit and dissemination of scientific research documents, whether they are published or not. The documents may come from teaching and research institutions in France or abroad, or from public or private research centers.
L'archive ouverte pluridisciplinaire HAL, est destinée au dépôt et à la diffusion de documents scientifiques de niveau recherche, publiés ou non, émanant des établissements d'enseignement et de recherche français ou étrangers, des laboratoires publics ou privés.

\section{(c)(1)}

Distributed under a Creative Commons Attribution| 4.0 International License 


\section{Adaptation of the Simple or Complex nature of $\mathrm{V} 1$ receptive fields to visual statistics}

\section{AUTHORS:}

Julien Fournier ${ }^{* 1}$, Cyril Monier ${ }^{1}$, Marc Pananceau ${ }^{1,2}$, and Yves Frégnac ${ }^{* 1}$

Unité de Neuroscience, Information et Complexité (CNRS-UNIC), Gif-sur-Yvette, France

${ }^{1}$ CNRS-UNIC, UPR CNRS 3293, Gif-sur-Yvette, F-91198;

${ }^{2}$ Université Paris-Sud, Orsay, F-91405.

${ }^{*}$ corresponding authors: J.F and Y.F.

Email: fournier@unic.cnrs-gif.fr, fregnac@unic.cnrs-gif.fr 


\section{ABSTRACT:}

Receptive fields (RFs) in primary visual cortex (V1) are categorized as Simple or Complex, depending on their spatial selectivity to stimulus contrast polarity. We studied the dependence of this classification on visual context by comparing, in the same cell, the synaptic responses to three classical RF mapping protocols: sparse noise, ternary dense noise and flashed Gabor noise. Intracellular recordings show that the relative weights of Simple-like and Complex-like RF components are scaled such as to make the same RF more Simple-like with dense noise and more Complex-like with sparse or Gabor noise stimulations. However, once these context-dependent RFs are convolved with the corresponding stimulus, the balance between Simple-like and Complex-like contributions to the synaptic responses appears invariant across input statistics. This normalization of the linear/nonlinear input ratio suggests a novel form of homeostatic control of V1 functional properties, optimizing the network nonlinearities to the statistical structure of the visual input. 


\section{INTRODUCTION}

The distinction between Simple and Complex cells ${ }^{1}$ relies on the degree of spatial segregation and linearity of responses to contrasts of opposite signs. Simple receptive fields (RFs) are classically considered as linear feature detectors, computing a weighted sum of the local contrast of the image $e^{1,2,3,4}$ whereas Complex RFs exhibit nonlinear spatial summation properties resulting in response invariance with regard to position or contrast polarity ${ }^{1,5-7}$.

Most extracellular surveys have reported V1 RFs with intermediate behaviors ${ }^{2,6,8}$ and intracellular recordings have shown that the separation between Simple and Complex cells hides a continuous distribution of synaptic inputs with regard to their degree of linearity ${ }^{9}$. In spite of the general acceptance that Simple and Complex cells correspond to distinct balance levels between linear and nonlinear contributions at the synaptic level ${ }^{10-12}$, few studies have investigated whether the functional expression of the Simple or Complex nature of V1 RFs depends or not, in the same cell, on the spatiotemporal statistics of the stimulus ${ }^{13,14}$. This may be surprising, since gain control mechanisms are known to ensure contrast-invariance of the cell selectivity ${ }^{15,16}$ and it is well established that adaptation to stimulus contrast does not have the same impact on the Simple-like and Complex-like components of $\mathrm{V} 1$ cell responses when assessed with drifting gratings ${ }^{17,18}$. Still, most studies of RF adaptation to visual statistics have focused either on linear RF components in Simple cells or on

nonlinear components in Complex cells ${ }^{19-23}$ but not on the differential adaptation of these two RF components in the same RF.

We address here this question by estimating synaptic and discharge fields of cat V1 cells recorded intracellularly in three white noise stimulus conditions: sparse noise $(\mathrm{SN})$, ternary dense noise (DN) and flashed Gabor noise (GBN). Our main finding is that the relative weights of the Simplelike and Complex-like components of the same RF adapt to the spatiotemporal statistics of the stimulus such that the respective contributions of these two functional components in the synaptic response remain in constant proportion across stimulus conditions once the RF is convolved with the visual stimulation sequence. We further show that this adaptation is not a straightforward scaling of the amplitudes of these RF components but results from differential changes in both their spatial extents and their temporal dynamics. These findings bring new evidences indicating that the functional properties of the V1 network, as measured from V1 RF estimates, are not fixed but adapt to the statistical properties of the visual input. 


\section{RESULTS}

Thirty-two neurons were recorded intracellularly in cat area 17 and their RFs were mapped with three protocols: sparse noise (SN), ternary dense noise (DN) and flashed Gabor noise (GBN) (Fig. 1a). Comparison across stimulus conditions was conducted at the subthreshold synaptic integration level (Vm RF) and at the spiking output level (Spk RF). We first focused our analysis on RF estimates obtained in the spatiotemporal domain ( $S N$ and $D N)$ and further extended the comparison to RFs estimated in the orientation domain (GBN).

Insert Figure 1 near here

\section{Stimulus dependence of $\mathrm{V} 1$ receptive field estimates}

Most previous RF studies using ternary white noise stimuli decomposed the cell input-output relationship into elementary responses to bright and dark stimuli ${ }^{1,6,9,24}$, namely ON and OFF subfields respectively. In this framework, the Simple or Complex nature of the RF was defined with different types of indexes measuring the degree of spatial segregation or antagonism between ON and OFF spatial profiles ${ }^{24}$. In studies using drifting gratings, Simple and Complex behaviors have been quantified according to the degree of linearity of the cell response with respect to the spatial phase of the stimulus at the preferred orientation and spatial frequency $y^{2,5,6,8}$. In the present study, we attempted to reconcile both classifications by switching from the classical ON OFF decomposition of white noise RF estimates to a Volterra RF expansion into first- $\left(h_{1 s t}\right)$ and second-order $\left(h_{2 n d}\right)$ kernels (Fig. 1b). The filter $h_{1 \text { st }}$ represents here the RF part which responds linearly with contrast polarity while the secondorder diagonal $\mathrm{h}_{2 \text { Diag }}$ pools nonlinear RF components independent of the contrast sign. The selectivity of this $h_{2 D i a g}$ component to stimulus features such as orientation and spatial frequency is defined by the off-diagonal elements of the second-order kernel ${ }^{5}$.

Due to the high dimensionality of this second-order kernel, we estimated off-diagonal interaction terms only in the DN condition, for subthreshold responses. On the one hand, the number of collected spikes was generally too small to proceed to a complete estimate of the spiking secondorder kernel. On the other hand, off-diagonal components are barely stimulated with sparse noise, making their estimation irrelevant in this stimulation context (Fig. 1c). While this limitation restricted

the comparison of RF estimates to the $\mathrm{h}_{1 \mathrm{st}}$ and $\mathrm{h}_{2 \mathrm{Diag}}$ filters, it was not critical with regard to our study: 
the estimation of the apparent Simple or Complex nature of the RF can be directly assessed from the balance between these two kernels without any knowledge of the feature selectivity underlying the Complex-like component $h_{2 \text { Diag. }}$ This truncated second-order Volterra expansion is computationally equivalent to the ON OFF decomposition: $h_{1 \text { st }}$ and $h_{2 \text { Diag }}$ kernels are strictly related to the difference and the sum respectively between ON and OFF kernels ${ }^{7}$. However, representing V1 RFs as $h_{1 s t}$ and $\mathrm{h}_{2 \mathrm{Diag}}$ components makes easier the distinction along both spatial and temporal RF dimensions between the net linear push-pull contribution, i.e. the Simple-like RF component in the strict sense, and the nonlinear contribution which responds in the same way to both contrast polarities, i.e. the Complex-like RF component.

Insert Figure 2 near here

Figure 2 illustrates this Volterra decomposition for two representative cells. Whether we consider the synaptic or the spiking RF estimates, the comparison between sparse and dense visual contexts reveals the same effect. $h_{1 s t}$ and $h_{2 \text { Diag }}$ waveforms were both substantially reduced in amplitude when switching from SN to DN conditions (Fig. 2b,d) but also exhibited systematic changes in their respective spatiotemporal organizations (Fig. 2a,c). While in the SN condition the RF of cell1 expresses a weak Simple-like component and a relatively large Complex-like contribution, DN stimulation of the same cell enhances the Simple-like contribution and reshapes the Complex-like component by shrinking its spatial and temporal extent. Similarly, in cell2, the RF switches from balanced Simple-like and Complex-like components in the SN condition to an almost complete suppression of the Complex-like contribution with DN. The same stimulus-dependent changes were observed in all cells we recorded: whatever the degree of linearity of the RF estimated in the sparse context, the balance between Simple-like and Complex-like RF components was modified such as to express stronger linear/Simple-like components $\left(h_{1 s t}\right)$ relative to the nonlinear/Complex-like components $\left(\mathrm{h}_{2 \mathrm{Diag}}\right)$ in the dense context (see also Supplementary Fig. 1). In the classical ON OFF perspective, the comparison of RF estimates between $\mathrm{SN}$ and $\mathrm{DN}$ conditions led to the same conclusion: ON and OFF subfields exhibited much smaller overlap and/or stronger antagonism in the dense than in the sparse noise condition (Supplementary Fig.2). 


\section{Receptive field Simpleness and gain controls}

To quantify the balance between Simple-like and Complex-like RF components in each stimulus condition, we defined a Simpleness Index $(S /)$, which measures the spatiotemporal energy of the linear $h_{1 s t}$ kernel relative to the sum of the $h_{1 s t}$ and $h_{2 \text { Diag }}$ spatiotemporal energies (see Online Methods). SI thus tends to "0" for Complex RF and converges to "1" for Simple RFs. Figures 3a and 3b show that DN stimuli result in a significant increase of RF Simpleness compared to SN, both at the subthreshold and spiking levels ( $P<<0.001$, paired Student's $t$ test). If one applies the $S I=0.5$ threshold as a distinction criterion, the synaptic RFs can be classified as Simple in $81 \%$ of cases in the DN context vs only $28 \%$ in the $\mathrm{SN}$ condition. A similar switch in RF type was found at the spiking level with $75 \%$ of Simple cells with DN vs 33\% with SN. Supplementary Figure 3 shows that the difference in $\mathrm{SI}$ trends observed between $\mathrm{SN}$ and $\mathrm{DN}$ conditions when comparing spike and $\mathrm{Vm}$-based measures is not due to a change in the spike threshold nonlinearity but rather to the impact of the half rectification of the $\mathrm{Vm}$ response on the read-out of the RF Simpleness.

An unexpected finding of our study is that a strikingly uniform behavior emerges from the various recorded cells when comparing the $S /$ values computed for dense and sparse conditions: all the data points, each representing one cell, are positioned along a continuous smooth curve in the bivariate correlation plot between DN and SN S/ values (Fig. 3a,b). We quantified the change in gain of the $h_{1 s t}$ and $h_{2 \text { Diag }} R F$ components by a gain factor (Gain ${ }_{S N / D N}$ ), defined as the ratio of the Euclidian norms of the kernel estimates when switching from SN to DN. Over the population, the Gain ${ }_{\text {SN/DN }}$ values measured on first-order synaptic components are consistent on average with an optimal gain control which would normalize the linear filter output relatively to the 8-fold difference between the standard deviations of luminance values in $\mathrm{SN}$ and DN conditions (Fig. 3c, Vm: mean $\mathrm{h}_{1 \mathrm{st}} \mathrm{Gain}_{\mathrm{SN} / \mathrm{DN}}=$ 7.75). For the Complex-like RF components $h_{2 \text { Diag }}$, the $G i_{S N / D N}$ values are all larger than for Simplelike components, both at the synaptic and the spiking level (Fig. 3c; Vm: mean $\boldsymbol{h}_{2 \text { Diag }}$ Gain $_{\mathrm{SN} / D N}=25.0$, $P<<0.001$, paired Student's $t$ test), in agreement with an increased linearity of the RFs in the DN context.

Insert Figure 3 near here 
Most remarkably, despite the large variability in $\mathrm{h}_{1 \mathrm{st}}$ Gain ${ }_{\mathrm{SN} / \mathrm{DN}}$, the gain controls affecting the Complex-like $\left(\mathrm{h}_{2 \mathrm{Diag}} \operatorname{Gain}_{\mathrm{SN} / \mathrm{DN}}=\beta\right)$ and Simple-like $\left(\mathrm{h}_{1 \mathrm{st}} \mathrm{Gain}_{\mathrm{SN} / \mathrm{DN}}=\alpha\right)$ RF synaptic components are related to each other according to a linear relationship over the cell population (Fig. 3c, slope $\left.k=\beta / \alpha=3.53, r^{2}=0.98, P<<0.01, n=30\right)$. A similar linear relationship was also observed at the spiking level, with a half slope value (Fig. $3 d$, slope $k=\beta / \alpha=1.81,11$ of $12, r^{2}=0.90, n=11$ ). This proportionality rule explains the smoothness of the global behavior observed in the Simpleness Index (blue curves in Fig. 3a,b, see Online Methods) and the best fit at the population level is given by a hyperbolic function parameterized by the slope of the regression (see Supplementary Note 2):

$$
S I_{D N}=\frac{S I_{S N}}{S I_{S N^{+}}\left(\frac{\alpha}{\beta}\right)^{2} \times\left(1-S I_{S N}\right)}
$$

For all cells, $h_{1 s t}$ and $h_{2 \text { Diag }}$ Gain $n_{S N / D N}$ values were larger than 1 , reflecting a systematic downscaling of the full RF from SN to DN conditions (see Supplementary Fig. 4). They also appeared negatively correlated with the $S /$ indexes, indicating that the more Simple the cell, the weaker the gain controls on Simple-like and Complex-like RF components when switching from sparse to dense noise (see Supplementary Fig. 5).

As illustrated by cell1 and cell2 (Fig. 2), these gain controls are not a straightforward rescaling of the kernel profiles but imply differential changes, at a synaptic level, in both their apparent spatial extent and their temporal dynamics across stimulus conditions: 1) spatially, the visuotopic extents of first-order RF components are significantly enlarged when switching from sparse to dense noise (Fig. 4a, left panel) whereas second-order diagonal subfields shrink conversely (Fig. 4a, right panel); 2) temporally, first-order RF components $\left(\mathrm{h}_{1 \mathrm{st}}\right)$ have a shorter latency in DN than in SN condition (Fig. $\mathbf{4 b}$, left panel) whereas the second-order RF components $h_{2 \text { Diag }}$ do not change in latency (Fig. $\mathbf{4 b}$, right panel) but reduce in duration (Fig. 4c, right panel). This late shortening suggests the implication of delayed inhibition reducing the propagation of nonlinear/Complex-like components in the dense noise regime and restricting their response integration time relative to the sparse noise condition.

\section{Insert Figure 4 near here}

The functional significance of these RF changes is confirmed by the ability of SN and DN subthreshold RFs to predict responses to new sequences of SN and DN stimuli (validation set of single trial data, see Online methods): even though, at the population level, SN and DN RFs only partially predicted responses corresponding to the same stimulus condition, they almost systematically 
failed to explain the response to the other stimulus class as well as the RF estimated in the same stimulus condition as the validation data set (see Supplementary Fig. 6; $P<<0.01$, paired Student's $t$ test).

\section{V1 receptive field Simpleness adapts to visual statistics}

To measure the balance between Simple-like and Complex-like synaptic contributions once the RF is convolved with the stimulus, we computed another Simpleness Index $\left(S I^{\star}\right)$, based on the reconstructed outputs of the first- and second-order kernel estimates (Fig. 5a, see Online Methods). This convolution results in a striking realignment of the Simpleness indexes: over the cell population, the $S I^{*}$ values are indistinguishable between $S N$ and DN (Fig. 5b, right panel), suggesting that the balance between Simple-like and Complex-like contributions remains unchanged in V1 cell synaptic activity, whether the visual test noise is sparse or dense.

In order to test a possible generalization to other visual input statistics, we measured the Simple or Complex nature of synaptic and spiking RFs using flashed Gabor noise (GBN) (for 20/32 cells, see Supplementary Fig. 7). The SI computed in the GBN condition reveals that synaptic (Fig. 5c, middle panel) and spiking RFs (see Supplementary Fig. 7c) are far more Complex with GBN than with DN (or, to a lesser extent, with $\mathrm{SN}$, data not shown). This result is consistent with our previous finding, since, for cortical cells, the Gabor noise protocol can be considered as a sparse stimulation in the Fourier domain. Accordingly, we found that no significant difference is noticeable between $S{ }^{*}$ values measured in the GBN and DN (Fig. 5c, right panel) or SN (data not shown) conditions, which reinforces and generalizes our hypothesis of a normalization of the Simpleness of the visually-evoked synaptic drive.

This realignment of the Simpleness indexes observed after convolving the RFs with the different stimulus sequences is visible even when considering only the $h_{2 D i a g}$ filter for reconstructing the Complex-like component of the DN response (see Supplementary Fig. 8). Therefore, this normalization cannot be simply explained by the recruitment of the off-diagonal terms of the secondorder kernel in the DN context; the stimulus dependent changes observed on the second-order diagonal $h_{2 \text { Diag }}$ contribute substantially to the invariance of the Simpleness of the reconstructed RF outputs across input statistics. 
Insert Figure 5 near here

The most sophisticated fits of V1 RFs in the literature rely on a convergence of multiple parallel linear subunits whose outputs are combined nonlinearly (parallel LN cascade model ${ }^{12}$ ). In this model architecture, the afferent filters can be considered as Simple-like or Complex-like RF components depending on the degree of sensitivity to contrast polarity along the stimulus feature dimension for which they are selective. To assess whether the stimulus dependent changes in RF Simpleness could arise trivially from mapping such second-order RF architectures with different stimulus statistics, we simulated non-adaptive RF models, made up of one Simple-like subunit in parallel with multiple linear filters whose outputs were squared (Fig. 6a). By imposing in graded ways the relative weights of the quadratic and the linear components, we synthesized a set of RF models which each expressed a distinct degree of Complex behavior. We simulated the responses to $\mathrm{SN}$, GBN and DN for each of these "non-adaptive" RF models and estimated the first- and second-order kernels, using the same decomposition as in our V1 cells. The SI measures obtained from these RF models did not differ between SN and DN (Fig. 6b, middle panel) or GBN and DN conditions (Fig. 6c, middle panel) which demonstrates that the effect we observed in our V1 cell population is unlikely to be due to any bias of our kernel estimates by the statistical properties of the visual input itself. These non-adaptive models further illustrate that, in the absence of adaptation of the RF Simpleness, the global synaptic responses evoked by dense stimuli are expected to express more Complex-like contributions than those evoked in sparse stimulation contexts: the $S I^{*}$ values computed directly from the RF model outputs reveal much stronger complexity in DN than in SN (Fig. 6b, right panel) or GBN contexts (Fig. 6c, right panel).

Taken together, these results strongly suggest that the stimulus dependent changes observed in V1 RFs Simpleness reflect a regulatory mechanism which compensates for the relative strength with which the stimulus recruits the Simple-like and Complex-like RF components, such as to maintain the Simpleness of the visually-evoked synaptic drive independent of changes in input statistics.

Insert Figure 6 near here

To get an insight into the time constants of this RF adaptation, we averaged across cells the synaptic responses evoked by each of these stimuli (see Supplementary Fig. 9). This mean response component, which reflects the dynamics of the Complex-like contributions averaged over the 
population, decays exponentially within the few first hundreds of milliseconds in SN ( $\tau=740 \mathrm{~ms}$ ), GBN ( $\tau=820 \mathrm{~ms}$ ) and DN ( $\tau=750 \mathrm{~ms}$ ) conditions. If we assume this decay is related to the adaptation of the Simple/Complex balance, its time constant suggests that this process is not instantaneous but fast enough to be still relevant regarding natural viewing condition dynamics ${ }^{25}$.

\section{Predictions by gain-control models}

In the perspective of our RF decomposition, the most straightforward model of adaptation of V1 RF Simpleness is to add two separate gain controls ( $\alpha$ and $\beta$ ) to the RF models depicted in Figure 6a, which would account respectively for the branch specific division of the Simple-like and Complexlike RF components when switching from SN to DN ("Differential" gain control model, DGC, Fig. 7a). However, we investigated whether our results could be explained by simpler adaptive models, in which the gain control process does not require any prior distinction between afferent RF components regarding the Simple or Complex nature of their contributions.

\section{Insert Figure 7 near here}

In the RF architecture depicted in Figure $\mathbf{6 a}$, a minimal hypothesis for adaptation of the cell response to the stimulus strength is to assume the existence of a post-NL gain control process $\gamma$, after the nonlinear filtering stage (post-NL), which changes the integrative properties of the model by normalizing the variance of the evoked response with regard to the increase in the stimulus power (GC1, Fig. 7d). Since this GC1 model affects equally the Simple-like and Complex-like afferent contributions, it is unable to explain any adaptation of the RF Simpleness between SN and DN (Fig. 7e, left panel) and the visually-evoked responses remain much more complex in DN than in SN conditions (Fig. 7e, right pane/). Still, one peculiar aspect of this model is that the gain factors measured on $h_{1 s t}$ and $h_{2 \text { Diag }}$ kernels are linearly related and inversely correlated with the degree of Simpleness of the RF, similar to what we found experimentally (Fig. 7c; see also Supplementary Fig. 4).

Another minimal adaptation hypothesis is to consider a pre-NL gain control process which rescales, before the nonlinear filtering stage (pre-NL), the gain of the afferent subunits with regard to the increase in the stimulus power ${ }^{26}$ (GC2, Fig. 7g). In this normalization model, all the linear filtering 
stages are divided by a single factor $g$ and the second-order RF components, which are related to the squared output nonlinearities, are scaled as $g^{2}$. Consequently, the RF Simpleness is expected to increase with the visual input strength. This type of model can account partially for our results: for a given RF structure, one can find a particular value $g^{*}$ for the divisive gain, corresponding to an underadaptation of the linear components, but for which the increase of RF Simpleness between SN and DN (Fig. 7h, left panel) compensates exactly for the strength with which these two stimuli recruit Simple-like and Complex-like RF components (Fig. 7h, right panel, see Supplementary Note 3). However, the GC2 model implies a quadratic relationship between the apparent scaling factors on Simple-like and Complex-like RF components (Fig. 7i), which is not consistent with our data. Moreover, in this model, different values of gain control $g$ necessarily lead to different degrees of adaptation of the RF Simpleness (Fig. 7 h). Thus, the GC2 model cannot explain both the diversity in the Gain ${ }_{S N / D N}$ values and the invariance of the $S I^{*}$ across stimulus conditions.

Interestingly, the combination of the two mechanisms, the pre-NL gain control $g^{*}$ with the post$N L$ gain control $\gamma(G C 3$, Fig. $7 \mathbf{j})$, appears to be sufficient to account for both the invariance of the $S I^{*}$

(Fig. 7k) between sparse and dense visual contexts and the linear relationship between $h_{1 s t}$ and $h_{2 D i a g}$ gain factors (Fig. 7I). This GC3 model illustrates that the adaptation of V1 RF Simpleness can be explained by gain control processes independent of the functional distinction between Simple-like and Complex-like RF components. Nevertheless, this conclusion holds only for the parameters depicted in Figure 7: none of these models is suitable for explaining a differential reorganization of Simple-like and Complex-like spatiotemporal profiles between sparse and dense visual contexts (Figs. 4 and 2). More realistic network architecture and/or gain controls with additional dynamic nonlinearities are needed to explain the spatiotemporal specificity of these kernel waveform changes.

\section{DISCUSSION}

Our intracellular study shows that the balance between Simple-like and Complex-like RF components depends on the statistics of the visual input, such as the same RF appears more Simplelike in dense than in sparse visual conditions. Stimulus dependent changes in the Simple or Complex behavior of V1 cells have already been reported in earlier studies using drifting grating stimuli, as a

function of stimulus contrast and RF surround recruitment ${ }^{17,13,27}$. However, this study is the first to 
show such clear changes in terms of spatiotemporal reorganizations of synaptic and discharge fields at the single cell level, interpretable as a coherent adaptive behavior at the population level. This stimulus dependence of V1 RF Simpleness does not exclude the possible existence of different classes of cells (in terms of afferent connectivity) in the cortical population. Our results rather suggest that it reflects an adaptation of the "effective connectivity" of the network ${ }^{28}$ to the statistical properties of the stimulus, making the balance between Simple-like and Complex-like synaptic influences $\left(S I^{*}\right)$ afferent to any cortical cell invariant from changes in visual input statistics. If one accepts the simplified view that the Simpleness Index reflects, for any given RF, the relative dominance of the local thalamic drive $^{29,30}$, it is likely that even in cells which receive direct geniculo-cortical afferents (in cat area 17, those in layer 4 and to a lesser extent layer 6$)^{31}$, the RF Simpleness adaptation remains in proportion with the low impact of the Complex-like RF contributions they might receive, as for the cells situated in the upper right corner of Figure $3 a$ (close to the point where $S I_{D N}=S I_{S N}=1.0$ ). The anatomical reconstruction of four cells labelled with biocytin in this study supports the view that whatever their rank in the thalamo-cortical layer hierarchy, all cortical cells are likely to exhibit an adaptation of their RF Simpleness with graded extent (see Supplementary Fig. 10). This conclusion is consistent with the previously described stimulus dependence of linear kernel estimates in superficial vs input layers $^{22,23}$. Our data further suggest the existence of an adaptation process which would result, across all cortical layers, in a rescaling of the Complex-like components in proportion with the gain control affecting the Simple-like components. The effects we describe are more detectable at the subthreshold than at the spiking level, where additional static nonlinearities may interfere with the global read-out of the connectivity rule (see Supplementary Fig. 3).

Our results must be interpreted in a functional perspective and several non-exclusive mechanisms can still be considered.

Our data do not completely exclude the contribution of a subcortical adaptation process which would make the LGN inputs to the cortex more nonlinear in sparse visual conditions than in dense visual contexts ${ }^{32,33}$. However, this interpretation is insufficient to account for the fact that the Gabor noise stimuli induced V1 RF changes which are consistent with the sparse nature of the stimulation in the orientation domain, whereas they likely corresponded to dense visual inputs for retinal or thalamic cells in view of the range of spatial frequencies used ${ }^{34}$. 
Interestingly, our simulations show that a simple gain control model (Fig. 7j) can partially reproduce the adaptation of V1 RFs Simpleness, provided there is a specific rescaling of the strength of the inputs to the network, combined with a gain control of the amplification performed by the downstream cell. Literally, it suggests that this adaptive behavior could result from the combination of 1) activity-dependent adaptation at the thalamic level, synaptic depression at thalamo-cortical synapses or any mechanism which reduces the impact of the feed-forward drive and 2) synaptic depression at cortico-cortical synapses, increased levels of intracortical inhibition, activity-dependent changes in cell intrinsic properties or any mechanism which reduces the intracortical amplification.

The increase of RF Simpleness in dense visual conditions could also be explained by a change in the balance between excitation and inhibition ${ }^{35}$ resulting in a suppression of the Complexlike synaptic components estimated in the $\mathrm{SN}$ context. In a generalized feed-forward perspective, this suppression could either come from an increase of push-pull inhibition ${ }^{36}$ or from the enhancement of an unselective Complex inhibition ${ }^{37,38,39}$ (see Supplementary Fig. 11) but in both cases, the apparent increase of RF linearity in the DN condition would result from the interplay between excitatory and inhibitory nonlinear inputs.

Another interpretation line would be to consider that the Simple or Complex nature of V1 RFs arises from a variable balance between feed-forward and lateral inputs ${ }^{40,10,11}$, the feed-forward drive providing the Simple-like component while the recurrent lateral connections convey Complex-like contributions. Accordingly, our results might be explained by the functional recruitment of lateral interactions in sparse stimulation conditions and by the decoupling of adjacent cortical columns in dense visual contexts (see Supplementary Fig. 11). This view is supported by recent results suggesting that the lateral propagation of activity between adjacent cortical units decreases substantially when increasing the contrast of the stimulation ${ }^{41}$. In view of our own results, the stimulus dependence of the lateral cortical interactions likely generalizes to other stimulus dimensions than the local contrast exclusively. Similar effects might be obtained by increasing the spatial or temporal density of the stimulus, the important parameter probably being the effective contrast along the stimulus feature dimensions for which the cell is selective ${ }^{42,43}$.

Regardless of the mechanisms underlying this adaptive behavior, one last important question is the relevance of this adaptation in natural viewing conditions. The synaptic normalization process we found could maintain in V1 the relative contribution of linear and nonlinear synaptic inputs invariant 
to dynamic changes in the local spatiotemporal context of the visual scene. During oculomotor exploration, depending on eye fixation location, a given RF would sample locally "sparser" or "denser" regions of a natural scene; synaptic normalization would allow the activated network to extract the same relative amount of information along the phase-sensitive and the phase-insensitive processing streams, thus ensuring the detection of the most relevant features of the visual scene independently of the local context in which they are embedded. This would improve information transmission by avoiding the over-representation of second-order correlations among cell population responses in dense visual contexts while increasing their detectability in sparse contexts, thus adapting the neuron's dynamic range to the level of correlation present in the visual input. We finally propose that the stimulus dependence of V1 RF Simpleness reflects a general rule of functional homeostasis common to many sensory systems ${ }^{44-47,33}$, which would ensure the adaptation of the network nonlinearities to ongoing changes in the statistical structure of the sensory input, according to optimal encoding principles ${ }^{48}$. 


\section{Acknowledgements}

We acknowledge the financial support of CNRS, the Agence Nationale de la Recherche (ANR: Natstats and V1-Complex) and EC contracts Facets (FP6-2004-IST-FETPI 15879) and Brain-i-nets (FP7-2009-ICT-FET 243914). We are thankful to Gérard Sadoc for his invaluable technical assistance in developing stimulation software and kernel analysis library tools. We thank Andrew Davison for comments and suggestions on the manuscript. We are thankful to Zoltan Kisvarday and Katelin Sari for their help in the biocytin labeling protocol.

\section{Author contributions}

This study is part of J.F.'s PhD thesis. The work plan was conceived by J.F., C.M. and Y.F. The experiments were performed by J.F, C.M. and M.P.. J.F. performed the data analysis and model simulations. J.F, C.M., and Y.F. wrote the paper. 


\section{FIGURE LEGENDS:}

\section{Figure 1: White noise stimuli and second-order Volterra RF decomposition}

(a) Example of single-trial intracellular responses evoked in the same cell (cell1) by sparse (SN, black), dense (DN, red) and gabor (GBN, grey) noise stimuli. The visual stimulation period is indicated by the horizontal black line. Spike amplitudes have been cut off at $-30 \mathrm{mV}$ to facilitate the comparison between membrane potential $(\mathrm{Vm})$ fluctuation dynamics. (b) First- and second-order Volterra kernels were estimated using a least-squares method. In this decomposition, the $h_{1 s t}$ kernel linearly filters the stimulus contrast variations and can be considered as the Simple-like part of the RF in the strict sense. In contrast, the second-order diagonal $\mathrm{h}_{2 \mathrm{Diag}}$ corresponds to the projection of the second-order RF nonlinearities in the first-order stimulus space, pooling RF components independent of the contrast sign, and can be considered as the Complex-like part of the RF. The feature selectivity underlying this

$h_{2 \text { Diag }}$ Complex-like component is provided by the off-diagonal terms of the second-order kernel $h_{2 n d}$. (c) Probability of stimulation $(\mathrm{P}(\mathrm{stim}))$ of the second-order kernel by $\mathrm{SN}$ (left) and $\mathrm{DN}$ (right). In contrast to DN stimuli, pixels are activated one at a time in SN condition. Consequently, off-diagonal components of the second-order kernel are barely stimulated by SN compared to the diagonal elements, making their estimation irrelevant in sparse stimulation contexts. 


\section{Figure 2: Stimulus dependence of Simple-like and Complex-like RF components}

(a) First-order kernel (left column, Simple-like, $\mathrm{h}_{1 \mathrm{st}}$ ) and second-order diagonal kernel (right column, Complex-like, $\mathrm{h}_{\text {2Diag }}$ ) of subthreshold $(\mathrm{Vm})$ and spiking $(\mathrm{Spk})$ receptive field estimates for a typical $\mathrm{V} 1$ cell (cell 1). Kernels are depicted as spatial (XY) and 2D spatiotemporal (YT) Z-scored maps. The XY spatial maps are shown for the lag time corresponding to their maximal spatial extent (indicated by the vertical black line in YT spatiotemporal profiles). The thin grey lines show the pixel size used for SN and DN. (b) Examples of elementary responses corresponding to positions indicated in the inset, overlaid over the shaded responsive area. Note the differences of scale between SN (black) and DN (red) waveforms, reflecting a divisive gain control of both Simple-like and Complex-like RF components when switching from SN to DN. (c-d) Same graphs as in (a-b) for another cell example (cell 2). 
Figure 3: RF Simpleness and gain control of Simple-like and Complex-like RF components

(a-b) Comparison over the population of recorded cells of the Simpleness Index (SI) measured from synaptic (a) or spiking (b) RF estimates between SN and DN conditions. All points lie above the identity line, showing that all RFs undergo a systematic change in the balance between Simple-like and Complex-like RF components such that they appear more Simple in the DN than in the SN condition. The data points corresponding to the example cells (shown in Fig. 2) have been circled. In the SN context, cell1 and cell2 subthreshold RFs exhibit S/ values of 0.08 and 0.56 respectively $(0.24$ and 0.46 at the spiking level), while in the DN condition, $S /$ values corresponding to the same cells increase to 0.32 and 0.90 respectively ( 0.53 and 0.77 at the spiking level). (c-d) Comparison between the Gain $n_{\text {SN/DN }}$ measured for Complex-like ( $h_{\text {2Diag }}$ Gain ${ }_{\text {SN/DN }}$ ) and Simple-like $\left(h_{1 s t}\right.$ Gain $\left._{\text {SN/DN }}\right)$ RF components, at the subthreshold (c) and spiking (d) levels. The gain factors affecting the Complex-like components are systematically higher and appear linearly related to the amplitude of the gain controls measured from the first-order components $h_{1 s t}$, except for two outliers (grey symbols) (blue regression lines, $V m$ : slope $=+3.53, r^{2}=0.98, P<<0.01, n=30 ;$ Spk: slope $=+1.81, r^{2}=0.90, P<<0.01, n=11$ ). The vertical dotted line indicates the value we would expect from perfectly adapting linear RF components: $h_{1 s t}$ Gain ${ }_{\text {SN/DN }}$ would correspond to the ratio of the SN and DN standard deviations of luminance values ( $\sim .16$, see Online Methods). 


\section{Figure 4: Spatiotemporal reconfiguration of Simple-like and Complex-like RF components}

(a) Comparison between DN and SN conditions of the maximal spatial extents of significant responses measured in Simple-like ( $\mathrm{h}_{1 \text { st }}$, left panel) and Complex-like ( $\mathrm{h}_{2 \mathrm{Diag}}$, right panel) RF components (units: visual degree of apparent diameter). While Simple-like RF components appear significantly larger in the DN than in the SN condition (paired Student's $t$ test, $P<0.01$ ), the Complex-like RF components are significantly shrunk (paired Student's $t$ test, $P \ll 0.01$ ). (b) Comparison of onset latencies of Simple-like $\left(\mathrm{h}_{1 \mathrm{st}}\right.$, left panel) or Complex-like ( $\mathrm{h}_{2 \text { Diag }}$, right panel) RF components between DN and SN conditions (units: ms). (c) Comparison of peak latencies of Simple-like $\left(\mathrm{h}_{1 \mathrm{st}}\right.$, left panel) or Complex-like ( $\mathrm{h}_{2 \text { Diag }}$, right panel) RF components between DN and SN conditions (units: $\mathrm{ms}$ ). 


\section{Figure 5: V1 RF Simpleness adapts to visual statistics}

(a) In each stimulus condition, the Simpleness was measured in two ways: 1) by the $S /$ index which compares the relative power of the Simple-like $\left(h_{1 s t}\right)$ and Complex-like $\left(h_{2 \text { Diag }}\right)$ components of RF estimates (RF, middle panels) and 2) by the $S I^{*}$ index which measures the balance between Simplelike and Complex-like synaptic contributions once the stimulus-dependent RFs have been convolved with the corresponding stimulus sequences ( $\mathrm{RF}^{*} \mathrm{Stim}$, right panels). In sparse stimulation conditions, since the pixels are activated one at a time, the nonlinear contributions conveyed by the off-diagonal terms of the $h_{2 n d}$ kernel have barely any weight in the response and the output of the $h_{2 \text { Diag }}$ filter provides an almost complete estimate of the Complex-like synaptic contributions. In contrast, in the DN condition, since multiple pixels are activated at the same time, the dynamics of the evoked Complex-like response also rely on the selectivity of the $h_{2 \text { Diag }}$ RF components to the spatiotemporal patterns which are presented. We thus computed the convolution of the stimulus with the full secondorder kernel estimate $h_{2 n d}$ to reconstruct the Complex-like synaptic contributions evoked by DN stimuli. (b) Comparison of Simpleness indexes between SN and DN conditions: middle panel, same graph as

in Figure 3a; right panel, comparison of the $S I^{*}$ indexes. The diagonal lines represent the identity relation. Note that over the population, the $S I^{*}$ values are much more aligned along the identity line than the SI values. (c) Same as in (b) but when comparing GBN and DN conditions. 


\section{Figure 6: Simpleness in non-adaptive RF models}

(a) Parallel LN cascade RF architecture in which linear filter outputs corresponding to different stimulus feature selectivities are passed through a second-order polynomial nonlinearity (one linear branch and several quadratic branches). In this model architecture, the linear component provides Simple-like contributions while the quadratic components contribute in a Complex-like manner to the cell response. By keeping the same RF structure while imposing the relative weights of these two types of afferent RF components, we simulated a set of RFs, each expressing a fixed degree of Simpleness, and simulated their responses to SN, GBN and DN stimulus sequences. (b) Middle panel: Comparison between $\mathrm{SI}$ measured for RFs estimated from the simulated $\mathrm{SN}$ and DN responses of the non-adaptive RF models depicted in (a). Right panel: comparison of the $S I^{*}$ (measured directly from the RF model outputs) between SN and DN conditions. (c) Same as in (b) but when comparing GBN and DN conditions. 


\section{Figure 7: Simpleness in gain control RF models}

(a) "Differential" gain control model (DGC): the adaptation of V1 RF Simpleness can be explained by adding two separate gain control processes $(\alpha$ and $\beta$ ) to the RF models depicted in Figure $6 \mathbf{a}$. These processes respectively divide Simple-like and Complex-like RF components when switching from SN to DN. (b): Same graph as in Figure 5b. (c): Same graph as in Figure 3c. The colors of the symbols correspond to three different ranges of value for the S/ values measured in the SN condition.

(d) "post-NL" gain control model (GC1): a gain control process $\gamma$ acts after the nonlinear filtering stage (post-NL) and normalizes the variance of the evoked response across stimulus conditions. (e) SI (left) and $S I^{*}$ (right) measured from GC1 RF model responses in SN and DN conditions. (f) SN/DN gain factors $\left(G a n_{S N / D N}\right)$ measured from the $h_{1 s t}$ and $h_{2 \text { Diag }}$ kernels estimated from the GC1 RF responses. Dark and light colors of the symbols indicate low and high values of $\gamma$ respectively.

(g) "pre-NL" gain control model (GC2): a gain control process $g$ acts before the nonlinear filtering stage (pre-NL) and results in a division of linear filter outputs by $g$ when switching from SN to DN, independently of the RF Simpleness. (h) $S I$ (left) and $S I^{*}$ (right) measured from GC2 RF model responses in $\mathrm{SN}$ and $\mathrm{DN}$ conditions. (i) $\mathrm{SN} / \mathrm{DN}$ gain factors (Gain $\mathrm{SN}_{\mathrm{N} / \mathrm{DN}}$ ) measured from the $\mathrm{h}_{1 \mathrm{st}}$ and $h_{2 \text { Diag }}$ kernels estimated from the GC2 RF responses. The purple curve indicates the quadratic relationship. Dark and light colors of the symbols indicate low and high values of $g$ respectively. $g^{*}$ corresponds to the value for which we observed a complete adaptation of the RF Simpleness between $\mathrm{SN}$ and DN conditions $\left(S I^{*}{ }_{\mathrm{DN}}=S I^{*}{ }_{\mathrm{SN}}\right)$.

(j) "Hybrid" gain control model (GC3): Combination of the GC1 and GC2 models (with $g=g^{*}$ ). (k) $S I$ (left) and $S I^{*}$ (right) measured from GC3 RF model responses in SN and DN conditions. (I) SN/DN gain factors $\left(\right.$ Gain $_{\text {SN/DN }}$ ) measured from the $h_{1 \text { st }}$ and $h_{2 D i a g}$ kernels estimated from the GC3 RF responses. The slope of the regression line (blue) corresponds to $g^{*}$. Note that this model is mathematically equivalent to the "differential" gain control model (a) considering $\alpha=g^{*} \times \gamma$ and $\beta=$

\footnotetext{
$g^{*^{2}} \times \gamma$. Dark and light colors of the symbols indicate low and high values of $\gamma$ respectively.
} 


\section{ONLINE METHODS:}

\section{Animal preparation and electrophysiological recordings}

Data presented here were obtained from anesthetized (alfatesin) and paralyzed cats, according to the American Physiological Society's Guiding Principles in the Care and Use of Animals. Animals used in these experiments have been bred in the Central CNRS Animal Care (French Agriculture Ministry Authorization: B91-272-105) under required veterinary and National Ethical Committee supervision. Intracellular electrodes were made from $1.5 \mathrm{~mm}$ wall-thickness borosilicate pipettes filled with a solution of $\mathrm{KMS}$ (potassium methyl sulfate), $2 \mathrm{M}$ and $\mathrm{KCl}, 4 \mathrm{mM}$. In some experiments we labeled intracellularly the cells we recorded by adding biocytin (1\%) to the intra-pipette solution. Electrode resistances were in the range 60 to $90 \mathrm{M} \Omega$. Recordings were performed using an Axoclamp 2A amplifier.

\section{Visual Stimulation}

Visual stimuli were generated using in-house software (Elphy, Gerard Sadoc, UNIC-CNRS ${ }^{\mathrm{TM}}$ ) and presented on a gamma-corrected monitor with a refresh rate of $150 \mathrm{~Hz}$ and a background luminance of $12 \mathrm{~cd} \cdot \mathrm{m}^{-2}$. Three kinds of white noise stimuli were presented in the same explored visual area: 1) two-dimensional ternary sparse noise (SN) which consisted of random sequences of nonoverlapping white $\left(23 \mathrm{~cd} . \mathrm{m}^{-2}\right)$ or black $\left(1 \mathrm{~cd} . \mathrm{m}^{-2}\right)$ squares, presented one at a time on a uniform luminance background $\left(12 \mathrm{~cd} . \mathrm{m}^{-2}\right)$, over a $10 \times 10$ grid (except for cell 2: 15*15); 2) two-dimensional ternary dense noise (DN) which consisted of random sequences of squares (same squares as in the corresponding SN condition) which could be either white $\left(23 \mathrm{~cd} . \mathrm{m}^{-2}\right)$, black $\left(1 \mathrm{~cd}^{-\mathrm{m}^{-2}}\right)$ or equal to the background (12 cd. $\mathrm{m}^{-2}$ ) with equal probability. 3) flashed Gabor noise (GBN) consisting of random sequences of flashed Gabor stimuli. Michelson's contrast was held constant at 0.5 and the spatial Gabor attenuation constant was $40 \%$ of the explored region dimensions. For each frame, orientation, spatial frequency and spatial phase were randomly chosen from a discrete uniform distribution consisting of six orientations ranging from $0^{\circ}$ to $150^{\circ}$, five spatial frequencies ranging from 0.2 to 1.1 cycles per degree, and four spatial phases ranging from 0 to $270^{\circ}$.

The seed used for initializing the random process of these three white noise stimuli was changed for each sequence (a necessary condition in sparse sequences to estimate the receptive 
field function). For each cell, visual stimuli were presented in an interleaved way, in the same region of the visual field and at the same frequency (frame duration: $13 \mathrm{~ms}$ for 15 cells, $26 \mathrm{~ms}$ for 12 cells, 33 ms for 4 cells and $56 \mathrm{~ms}$ for 1 cell). We adapted the size of the explored visual area to the apparent spatial extent of the RF. Still, the number of pixels covering the significant RF area (SN and DN conditions) could vary from cell to cell (from 4 to 54 pixels) and was on average 20 pixels.

Note that while SN and DN stimuli exhibited the same elementary contrast steps (c), they differed strongly in spatiotemporal statistics. In the SN condition, as pixels are activated only one at a time, the power of the stimulus $\left(P^{2}\right)$ is very low $\left(P^{2}=2 / 200 \times c^{2}\right.$ for a $10 \times 10$ simulation grid). This is in contrast with the DN condition where the three luminance states are on average equally represented in each frame $\left(P^{2}=2 / 3 \times c^{2}\right)$. These differences between sparse and dense stimulation regimes give a ratio between their respective standard deviations of luminance values of

$$
\sqrt{\frac{2 / 3}{2 / 200}} \sim 8.16 .
$$

\section{Second-order Volterra kernel estimation}

Action potentials, when present, were automatically filtered out from synaptic fluctuations offline. First- and second-order RF components were estimated using a least-squares method, considering either the spiking response or the subthreshold fluctuations. This estimation consisted of solving the system of equations corresponding to the Volterra series expansion(Eq.1):

$$
\begin{aligned}
R(t)=h_{0}+ & \sum_{x, y, \tau} h_{1 s t}(x, y, \tau) \times S(x, y, t-\tau) \\
& +\sum_{x_{1}, y_{1}, \tau_{1} x_{2}, y_{2}, \tau_{2}} h_{2 n d}\left(x_{1}, y_{1}, \tau_{1}, x_{2}, y_{2}, \tau_{2}\right) \times S\left(x_{1}, y_{1}, t-\tau_{1}\right) \times S\left(x_{2}, y_{2}, t-\tau_{2}\right)
\end{aligned}
$$

Where $R(t)$ is the cell response recorded at time t, sampled with a 1 ms-resolution; $S$, the stimulus input vector (see Supplementary note 1 ) and $h_{0}, h_{1 s t}, h_{2 n d}$ correspond respectively to the zero-, firstand second-order Volterra kernels.

The kernels estimated from responses to flashed Gabor noise obeyed the same formalism as in the spatiotemporal domain except that $h_{1 s t}$ and $h_{2 n d}$ were functions of position in the Fourier space. In this condition, contrast of opposite signs corresponded to identical Gabor stimuli but with spatial phases separated by $180^{\circ}$. 
Note that due to the sparse statistical structure of SN and GBN stimuli, the off-diagonal elements of the $h_{2 n d}$ kernel are barely stimulated, which means that the estimation of the second-order RF components comes down to the estimation of its diagonal elements $h_{2 \text { Diag }}$ $\left(\mathrm{h}_{2 \text { Diag }}(x, y, \tau)=\mathrm{h}_{2 n d}(x, y, \tau, x, y, \tau)\right)$. Consequently, in these sparse stimulation conditions, the secondorder Volterra decomposition (Eq.1) can be simplified as:

$$
\left.\mathrm{R}(\mathrm{t})=\mathrm{h}_{0}+\sum_{\mathrm{x}, \mathrm{y}, \tau} \mathrm{h}_{1 \mathrm{st}}(\mathrm{x}, \mathrm{y}, \tau) \times S(\mathrm{x}, \mathrm{y}, \mathrm{t}-\tau)+\sum_{\mathrm{x}, \mathrm{y}, \tau} \mathrm{h}_{2 \mathrm{Diag}}(\mathrm{x}, \mathrm{y}, \tau) \times S(\mathrm{x}, \mathrm{y}, \mathrm{t}-\tau)^{2} \quad \text { (Eq. } 2\right)
$$

In contrast, in the DN condition, the full second-order kernel (diagonal and off-diagonal elements) is stimulated such that it is possible to estimate it completely. Still, due to the high dimensionality of this functional space, the estimation of the off-diagonal elements is constrained by the recording length. While the number of collected spikes was generally too small to compute this estimate, our voltage records allowed us to proceed to the estimation of the off-diagonal elements of the second-order kernel for all cells. In order to keep a ratio of at least 1:5 between the number of kernel parameters and the number of data points, we had to restrict spatially and temporally the estimation of these off-diagonal terms. Nonetheless, despite this constraint, we were able, for all cells, to cover in the off-diagonal space the elements corresponding to the full spatiotemporal extent of the significant responses estimated in the $h_{2 \text { Diag }}$ component. Note that the estimation of the $h_{2 \text { Diag }}$ kernel parameters was unchanged whether we considered the estimation of the off-diagonal elements of the second-order kernel or not.

\section{Variance of kernel estimates}

The statistical significance of kernel parameters was computed as spatiotemporal z-score maps in which each kernel parameter (corresponding to a particular spatiotemporal position) was divided by the standard deviation of the kernel error calculated from the parameter covariance matrix. A major benefit of using least-squares methods is that it provides a direct theoretical expression of the confidence bounds to take into consideration for kernel parameters ${ }^{49}$. If we assume that the corrupting noise in the output is a stimulus-independent Gaussian process with zero-mean and variance $\sigma^{2}$, the variance of each kernel parameter is given by the corresponding elements on the diagonal of the parameter covariance matrix $C_{\theta}$, which is computed from the inverse of the stimulus covariance matrix $\mathrm{H}$ (Hessian matrix) and an unbiased estimate of the residual variance $\sigma^{2}$ res: 


$$
C_{\theta}=\sigma_{\text {res }}^{2} \times H^{-1}(E q .3)
$$

where

$$
\sigma_{r e s}^{2}=\frac{1}{N-M} \times \sum_{\mathrm{t}}(\mathrm{R}(\mathrm{t})-\widehat{\mathrm{R}}(\mathrm{t}))^{2} \quad(E q .4)
$$

with $N$ being the number of time bins; $M$, the number of kernel parameters and $\widehat{R}(t)$, the response output reconstructed from the estimated kernels.

\section{Variance of the kernels output}

Since the kernel outputs are linearly related to their parameters, the variance of the error of the convolution product at time $t$ between the stimulus sequence and the kernel $\left(\left(\mathrm{h}_{1 \mathrm{st}} * S\right)_{(\mathrm{t})}\right.$ or $\left.\left(\mathrm{h}_{\text {2nd }} * S\right)_{(\mathrm{t})}\right)$ can be deduced from the parameter covariance matrix $C_{\theta}{ }^{49}$.

$$
\sigma_{(h * S)_{(t)}}^{2}=U_{h}(t)^{T} \times C_{\theta} \times U_{h}(t) \quad(E q .5)
$$

where $U_{h}(t)$ is a vector which contains the appropriate stimulus elements (i.e. $S(x, y, t-\tau)$ or $\left.S\left(\mathrm{x}_{1}, \mathrm{y}_{1}, \mathrm{t}-\tau_{1}\right) \times S\left(\mathrm{x}_{2}, \mathrm{y}_{2}, \mathrm{t}-\tau_{2}\right)\right)$ in positions corresponding to the parameters of the considered kernel and is zero elsewhere.

\section{Quantification of Kernel estimates}

In this study, all kernel quantifications were based on the total duration of the impulse responses instead of selecting one particular time epoch (such as when the RF amplitude or spatial extent is maximal). To avoid any contamination of our measurements by the residual noise of our kernel estimates, we only considered spatiotemporal positions which were statistically significant (zscore $\geq 2.33$, i.e. $P \leq 0.01)$. The Simpleness Index $S /$ was defined as follows:

$$
S I=\frac{\sum_{x, y, \tau} h_{1 s t}(x, y, \tau)^{2}}{\sum_{x, y, \tau} h_{1 s t}(x, y, \tau)^{2}+\sum_{x, y, \tau} h_{2 \operatorname{Diag}}(x, y, \tau)^{2}} \quad(E q .6)
$$

The relationship between DN and SN S/ values (Fig. 3a and $\mathbf{3 b}$ ) was fitted by the following equation:

$$
S I_{\mathrm{DN}}=\frac{S \mathrm{I}_{\mathrm{SN}}}{\mathrm{SI}_{\mathrm{SN}}+\left(\frac{1}{k}\right)^{2} \times\left(1-\mathrm{SI}_{\mathrm{SN}}\right)} \quad(E q .7)
$$


where $k$ is a constant parameter over the cell population (see Supplementary Note 2).

The gain factor Gain ${ }_{\mathrm{SN} / \mathrm{DN}}$ between $\mathrm{SN}$ and $\mathrm{DN}$ kernels was defined as the ratio of the kernel Euclidian norms associated with the different stimulus conditions, considering the kernels as spatiotemporal vectors:

$$
\mathrm{h}_{\text {Gain }} \text { SN/DN }=\frac{\sqrt{\sum_{x, y, \tau} \mathrm{h}^{S N}(\mathrm{x}, \mathrm{y}, \tau)^{2}}}{\sqrt{\sum_{x, y, \tau} \mathrm{h}^{D N}(\mathrm{x}, \mathrm{y}, \tau)^{2}}}(E q .8)
$$

The Simpleness Index, $S I^{*}$, which measures the strength of the Simple-like and Complex-like components of the synaptic response, was computed from the convolution products between the visual stimulus and the first-order $\left(h_{1 s t}\right)$ or second-order $\left(h_{2 n d}\right)$ kernels, considering only the components of the kernel output which were statistically significant (z-score $\geq 2.33$, i.e. $P \leq 0.01)$ :

$$
S I^{*}=\frac{\sum_{\mathrm{t}}\left(\mathrm{h}_{1 \mathrm{st}} * S_{(\mathrm{t})}\right)^{2}}{\sum_{\mathrm{t}}\left(\mathrm{h}_{1 \mathrm{st}} * S_{(\mathrm{t})}\right)^{2}+\sum_{\mathrm{t}}\left(\mathrm{h}_{2 \mathrm{nd}} * S_{(\mathrm{t})}\right)^{2}}
$$

where the $*$ symbol denotes the convolution product between the first- or second- order kernel and the stimulus sequence (see $E q .1$ ).

Note that in $\mathrm{SN}$ and GBN conditions, since the $h_{2 n d}$ kernel comes down to its diagonal elements $h_{2 \text { Diag }}$, the expression of $S I^{*}$ is equivalent to:

$$
S I^{*}=\frac{\sum_{\mathrm{t}}\left(\mathrm{h}_{1 \mathrm{st}} * S_{(\mathrm{t})}\right)^{2}}{\sum_{\mathrm{t}}\left(\mathrm{h}_{1 \mathrm{st}} * S_{(\mathrm{t})}\right)^{2}+\sum_{\mathrm{t}}\left(\mathrm{h}_{2 \text { Diag }} * S_{(\mathrm{t})}\right)^{2}} \quad(E q .10)
$$

Areas delimited by contours of $99 \%$-significant responses (z-score $\geq 2.33$, i.e. $P \leq 0.01)$ were measured for each kernel on spatially smoothed versions of their respective z-score maps and plotted over time. The maximal spatial extent of these contours (measured by their equivalent apparent diameter expressed in degrees of visual angle, Sig. spatial extent) as well as the timing of this maximum (Peak latency) was measured for each kernel to be compared between conditions. The onset latency of each kernel was derived from the contour area measurement. The initial radial growth of the significant responsive area was generally constant as a function of time and we computed the onset time in the following way: we defined an area threshold corresponding to half of the maximum and went backwards in time until the time derivative of the $\mathrm{RF}$ area fell below $10 \%$ of the derivative 
calculated at half-amplitude, for five continuous time steps $(5 \mathrm{~ms})$. This measure thus reflects the latency of the earliest response which initiates the progressive build-up of the full RF domain. We found much more reliable measurements using this method than with methods based on the derivative of the kernel waveforms.

\section{Predictive power of receptive field estimates}

We assessed the ability of SN and DN RF estimates to predict responses to new data sets which were not used for estimating the kernels. RF estimates were first convolved with the validation stimulus sequence according to equation $E q .1$ and rescaled according to the best linear coefficient (in the least squares sense) to avoid any failure of prediction just due to a static change in the linear gain. Predicted and recorded responses were low-pass filtered at $75 \mathrm{~Hz}$ and binned at 1-ms resolution. We finally quantified the accuracy of the prediction by measuring 1) the Predictive Power, defined as the percentage of variance of the cell response explained by the prediction and 2) the Predictive correlation, defined as the Pearson's correlation coefficient between measured and predicted responses:

$$
\begin{gathered}
\text { Predictive Power }=100 \times\left(1-\frac{\sum\left(V_{m}(t)-\widehat{V}_{m}(t)\right)^{2}}{\sum\left(V_{m}(t)-\left\langle V_{m}\right\rangle\right)^{2}}\right)(E q .11) \\
\text { Predictive correlation }=\frac{\sum\left(\left(V_{m}(t)-\left\langle V_{m}\right\rangle\right) \times\left(\widehat{V}_{m}(t)-\left\langle\widehat{V}_{m}\right\rangle\right)\right)}{\sqrt{\sum\left(V_{m}(t)-\left\langle V_{m}\right\rangle\right)^{2} \times \sum\left(\widehat{V}_{m}(t)-\left\langle\widehat{V}_{m}\right\rangle\right)^{2}}}(E q .12)
\end{gathered}
$$

where $\widehat{V}_{m}$ denotes the response predicted by the RF estimates, $V_{m}$ the recorded cell response and \langle\rangle , the mean response level.

To predict the response to the same stimulus condition as the one used for estimating the RF, we considered $95 \%$ of the total recording length for kernel estimation, computed the prediction on the $5 \%$ left with the estimated kernels and repeated this procedure until we completed the prediction of the full response.

Note that in order to have the longest recording duration for estimating the second-order kernels, we only recorded single trial responses (the seed of the white noise was changed every trial). Therefore, since the actual response variance also contains a certain amount of noise (which would have been reduced by averaging, had we used the same seed for all trials), our measurements of predictive 
power correspond to an underestimate of the true ability of RF estimates to predict the stimulus locked response of the validation data set.

\section{Receptive field simulations}

Simulated RFs were made up of a parallel bank of linear filters (Fig. 6a), shaped as a sum of two or three non-overlapping Gaussian zones with alternating polarities, so that they looked like typical Simple cell receptive fields. Each filter was then convolved with SN, GBN or DN sequences and their outputs were passed through a multidimensional second-order polynomial in which one of the filter outputs was linearly transformed while the others were squared. In this static nonlinearity, the strength of the first-order coefficient relative to that of the quadratic components thus influenced the Simple or Complex nature of the full RF model. This allowed us to define, in a graded way, several RF models with properties intermediate between purely Complex and purely Simple behaviors and whose degree of linearity remained the same across stimulus conditions. In addition to these non-adaptive RF models, we also simulated adaptive filter banks which were strictly identical to those simulated in Figure 6 but included gain control processes (Fig. $\mathbf{7 d}, \mathbf{7 g}$ and $\mathbf{7 j}$ ). In the GC1 RF models, the variance of the global response evoked by the stimulus sequence was forced to stay constant across stimulus conditions, by scaling the filter bank output after the nonlinear filtering stage with the appropriate factor $\gamma$. In the GC2 RF models, the gain of all linear subunits was divided by a constant factor $g$ when switching from SN to DN, regardless of the degree of linearity of the RF. Three different values of $g$ were tested: 1) $g=8.16$, corresponding to a full adaptation of the linear outputs to the change in stimulus energy; 2) $g=5$, 3) $g=g^{\star}=3.42$, corresponding to a full adaptation of the Simpleness of the RF model output between SN and DN $\left(S I_{D N}^{*}=S I_{S N}^{*}\right)$. Finally, in the GC3 RF models, we combined the division of the linear subunits by a factor corresponding to a complete adaptation of the $S I^{*}\left(g=g^{*}\right)$ and the normalization of the variance of the filter bank output across stimulus conditions. 


\section{REFERENCES}

1. Hubel, D. \& Wiesel, T. Receptive fields, binocular interaction and functional architecture in the cat s visual cortex. J Physiol 160, 106-154(1962).

2. Movshon, J., Thompson, I. \& Tolhurst, D. Spatial summation in the receptive fields of simple cells in the cat s striate cortex. J Physiol 283, 53-77(1978).

3. DeAngelis, G.C., Ohzawa, I. \& Freeman, R.D. Spatiotemporal organization of simple-cell receptive fields in the cat $s$ striate cortex. II. Linearity of temporal and spatial summation. $J$ Neurophysiol 69, 1118-1135(1993).

4. Carandini, M. et al. Do we know what the early visual system does? J Neurosci 25, 1057710597(2005).

5. Movshon, J., Thompson, I. \& Tolhurst, D. Receptive field organization of complex cells in the cat s striate cortex. J Physiol 283, 79-99(1978).

6. Dean, A.F. \& Tolhurst, D.J. On the distinctness of simple and complex cells in the visual cortex of the cat. J Physiol 344, 305-325(1983).

7. Emerson, R.C. et al. Nonlinear directionally selective subunits in complex cells of cat striate cortex. J Neurophysiol 58, 33-65(1987).

8. Skottun, B.C. et al. Classifying simple and complex cells on the basis of response modulation. Vision Res 31, 1079-1086(1991).

9. Priebe, N.J. et al. The contribution of spike threshold to the dichotomy of cortical simple and complex cells. Nat Neurosci 7, 1113-1122(2004).

10. Chance, F.S., Nelson, S.B. \& Abbott, L.F. Complex cells as cortically amplified simple cells. Nat Neurosci 2, 277-282(1999).

11. Tao, L. et al. An egalitarian network model for the emergence of simple and complex cells in visual cortex. PNAS 101, 366-371(2004).

12. Rust, N.C. et al. Spatiotemporal elements of macaque v1 receptive fields. Neuron 46, 945956(2005).

13. Bardy, C. et al. "Simplification" of responses of complex cells in cat striate cortex: suppressive surrounds and "feedback" inactivation. J Physiol 574, 731-750(2006).

14. Victor, J.D. et al. Responses of V1 Neurons to Two-Dimensional Hermite Functions. $J$ Neurophysiol 95, 379-400(2006).

15. Albrecht, D.G., Farrar, S.B. \& Hamilton, D.B. Spatial contrast adaptation characteristics of neurones recorded in the cat $\mathrm{s}$ visual cortex. J Physiol 347, 713-739(1984).

16. Ohzawa, I., Sclar, G. \& Freeman, R.D. Contrast gain control in the cat s visual system. $J$ Neurophysiol 54, 651-667(1985).

17. Carandini, M. \& Ferster, D. A tonic hyperpolarization underlying contrast adaptation in cat visual cortex. Science 276, 949-952(1997). 
18. Sanchez-Vives, M.V., Nowak, L.G. \& McCormick, D.A. Membrane mechanisms underlying contrast adaptation in cat area 17 in vivo. J Neurosci 20, 4267-4285(2000).

19. Sharpee, T.O. et al. Adaptive filtering enhances information transmission in visual cortex. Nature 439, 936-942(2006).

20. Felsen, G. et al. Cortical sensitivity to visual features in natural scenes. PLoS Biol 3, e342(2005).

21. David, S.V., Vinje, W.E. \& Gallant, J.L. Natural stimulus statistics alter the receptive field structure of v1 neurons. J Neurosci 24, 6991-7006(2004).

22. Yeh, C.I. et al. Stimulus ensemble and cortical layer determine V1 spatial receptive fields. Proc Natl Acad Sci U S A 106, 14652(2009).

23. Victor, J.D. et al. Laminar and Orientation-Dependent Characteristics of Spatial Nonlinearities : Implications for the Computational Architecture of Visual Cortex. J Neurophysiol 102, 34143432(2009).

24. Mata, M.L. \& Ringach, D.L. Spatial Overlap of ON and OFF Subregions and Its Relation to Response Modulation Ratio in Macaque Primary Visual. J Neurophysiol 93, 919-928(2005).

25. Stryker, M. \& Blakemore, C. Saccadic and disjunctive eye movements in cats. Vision Res 12, 2005-13(1972).

26. Carandini, M. \& Heeger, D.J. Summation and division by neurons in primate visual cortex. Science 264, 1333-1336(1994).

27. Crowder, N.A. et al. Complex cells increase their phase sensitivity at low contrasts and following adaptation. J Neurophysiol 98, 1155-1166(2007).

28. Aertsen, A.M. et al. Dynamics of neuronal firing correlation: modulation of "effective connectivity". J Neurophysiol 61, 900-17(1989).

29. Martinez, L.M. \& Alonso, J.M. Construction of complex receptive fields in cat primary visual cortex. Neuron 32, 515-525(2001).

30. Martinez, L.M. et al. Receptive field structure varies with layer in the primary visual cortex. Nat Neurosci 8, 372-379(2005).

31. LeVay, S. \& Gilbert, C.D. Laminar patterns of geniculocortical projection in the cat. Brain Res 113, 1-19(1976).

32. Lesica, N.A. et al. Adaptation to stimulus contrast and correlations during natural visual stimulation. Neuron 55, 479-491(2007).

33. Smirnakis, S.M. et al. Adaptation of retinal processing to image contrast and spatial scale. Nature 386, 69-73(1997).

34. So, Y.T. \& Shapley, R. Spatial tuning of cells in and around lateral geniculate nucleus of the cat: $X$ and $Y$ relay cells and perigeniculate interneurons. $J$ Neurophysiol 45, 107-120(1981).

35. Sillito, A.M. The contribution of inhibitory mechanisms to the receptive field properties of neurones in the striate cortex of the cat. J Physiol 250, 305-329(1975). 
36. Troyer, T.W. et al. Contrast-invariant orientation tuning in cat visual cortex: thalamocortical input tuning and correlation-based intracortical connectivity. J. Neurosci. 18, 5908-5927(1998).

37. Lauritzen, T.Z. \& Miller, K.D. Different roles for simple-cell and complex-cell inhibition in V1. J Neurosci 23, 10201-10213(2003).

38. Hirsch, J. et al. Functionally distinct inhibitory neurons at the first stage of visual cortical processing. Nat Neurosci 6, 1300-1308(2003).

39. Liu, B.H. et al. Visual receptive field structure of cortical inhibitory neurons revealed by twophoton imaging guided recording. J Neurosci 29, 10520-10532(2009).

40. Debanne, D., Shulz, D.E. \& Fregnac, Y. Activity-dependent regulation of "on" and "off" responses in cat visual cortical receptive fields. J Physiol 508 (Pt 2), 523-548(1998).

41. Nauhaus, I. et al. Stimulus contrast modulates functional connectivity in visual cortex. Nat Neurosci 12, 70-76(2009).

42. Müller, J.R. et al. Rapid adaptation in visual cortex to the structure of images. Science 285, 1405-1408(1999).

43. Felsen, G. et al. Dynamic modification of cortical orientation tuning mediated by recurrent connections. Neuron 36, 945-954(2002).

44. Nagel, K.I. \& Doupe, A.J. Temporal processing and adaptation in the songbird auditory forebrain. Neuron 51, 845-859(2006).

45. Maravall, M. et al. Shifts in Coding Properties and Maintenance of Information Transmission during Adaptation in Barrel Cortex. PLoS Biol 5, e19(2007).

46. Higley, M.J. \& Contreras, D. Frequency adaptation modulates spatial integration of sensory responses in the rat whisker system. J Neurophysio/ 97, 3819-24(2007).

47. Brenner, N., Bialek, W. \& Ruyter van Steveninck, R. de Adaptive rescaling maximizes information transmission. Neuron 26, 695-702(2000).

48. Wainwright, M.J. Visual adaptation as optimal information transmission. Vision Res 39, 3960 3974(1999).

49. Westwick, D.T. \& Kearney, R.E. Identification of nonlinear physiological systems. (IEEE Press: Piscataway: Wiley-IEEE, 2003). 


\section{a.}
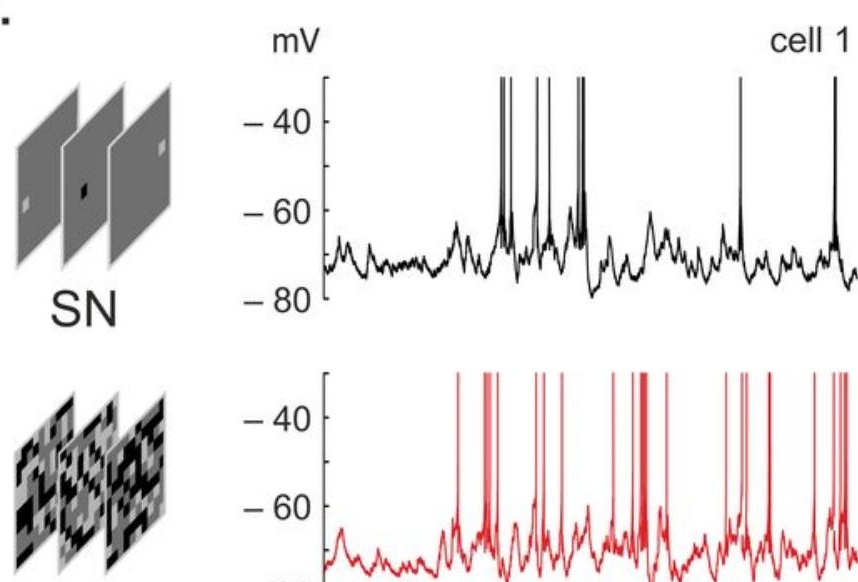

DN

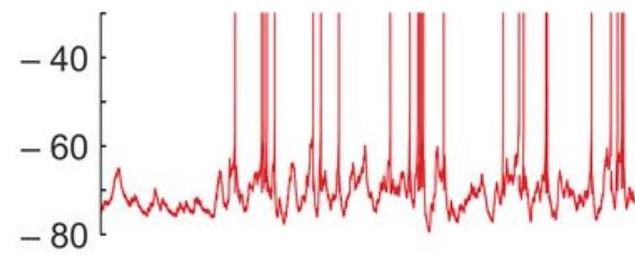

GBN
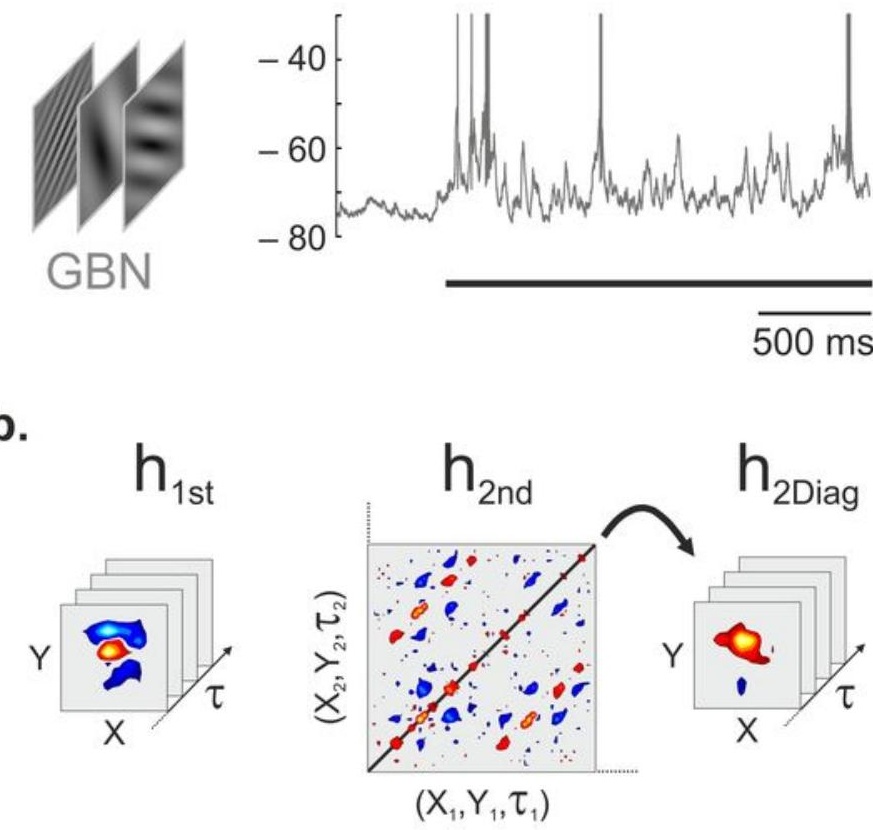

c.
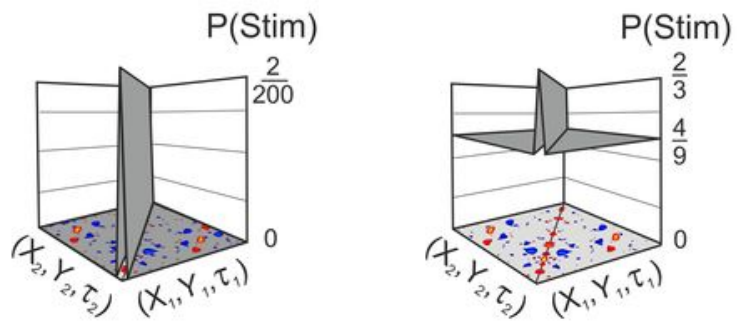

SN

DN 
a. cell 1

$\mathrm{h}_{1 \mathrm{st}}$

$h_{\text {2Diag }}$

$\mathrm{Vm}$

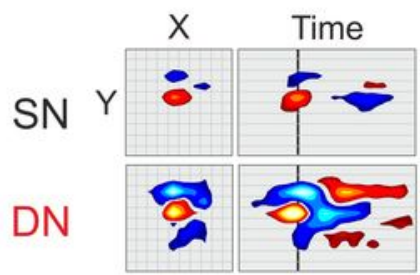

Spk

SN

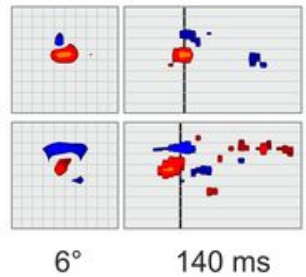

cell 2

$\mathrm{h}_{1 \mathrm{st}}$

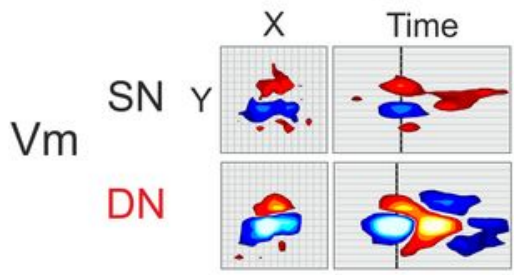

SN

Spk

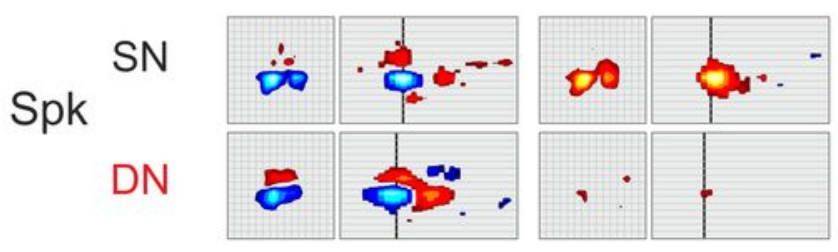

$140 \mathrm{~ms}$

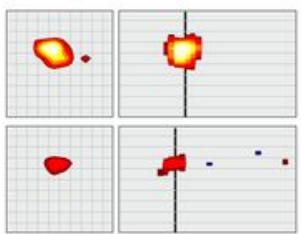

$\mathrm{h}_{\text {2Diag }}$

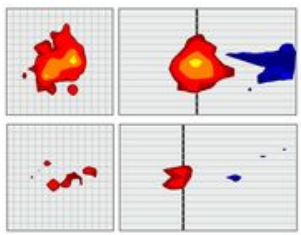

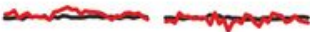

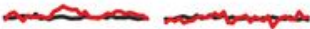

aran

$120 \mathrm{~Hz}$

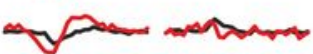
$140 \mathrm{~ms}$
$11 \mathrm{mV}$

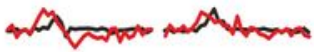

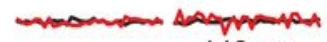

d.

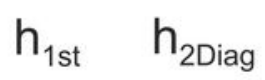

$+$

7.50

1.64

- 1.64 - $\begin{aligned} & 2.33 \\ & 7.50\end{aligned}$ $>10.0$

S 

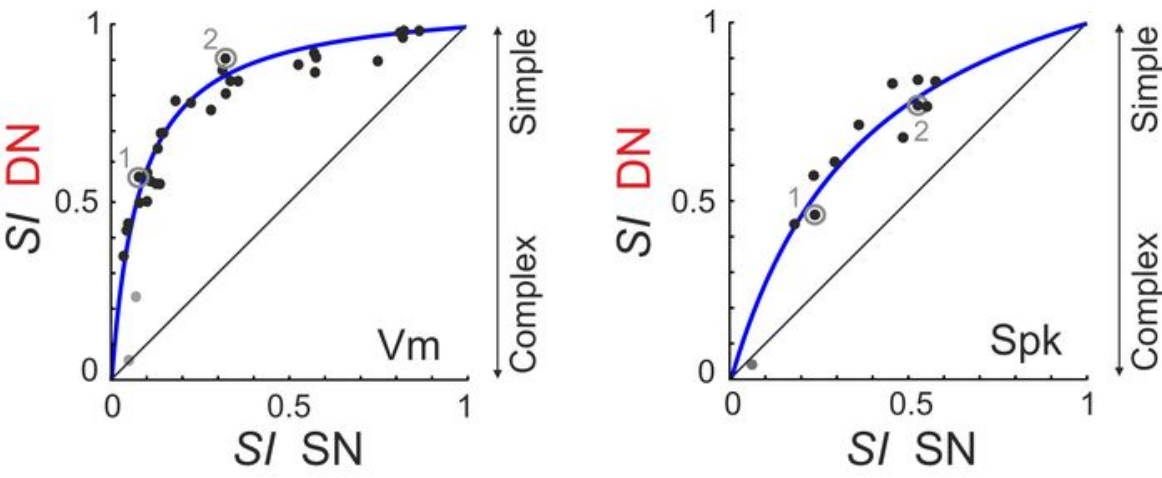

c.

d.
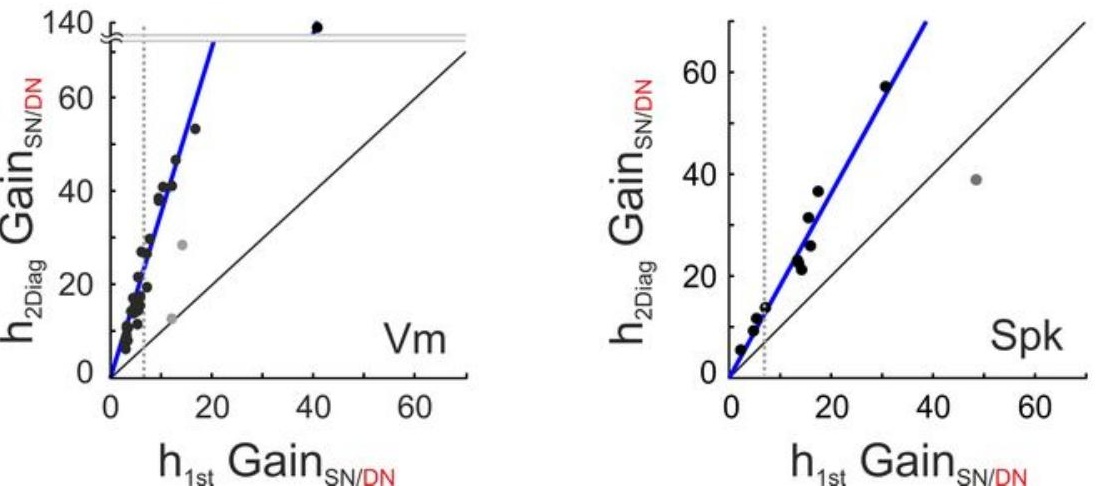

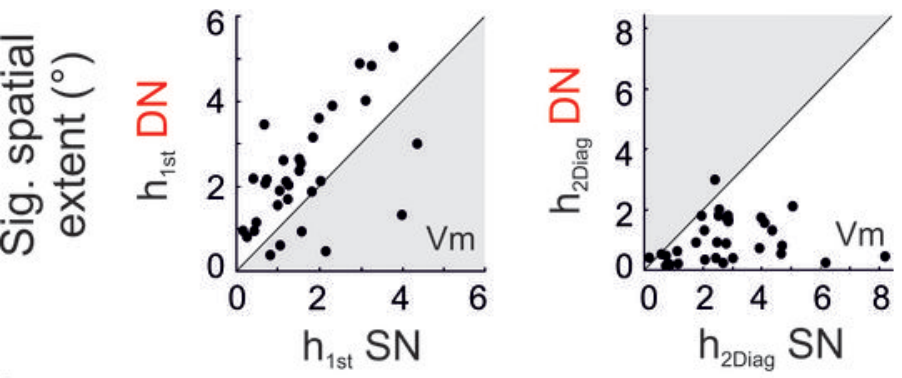

b.
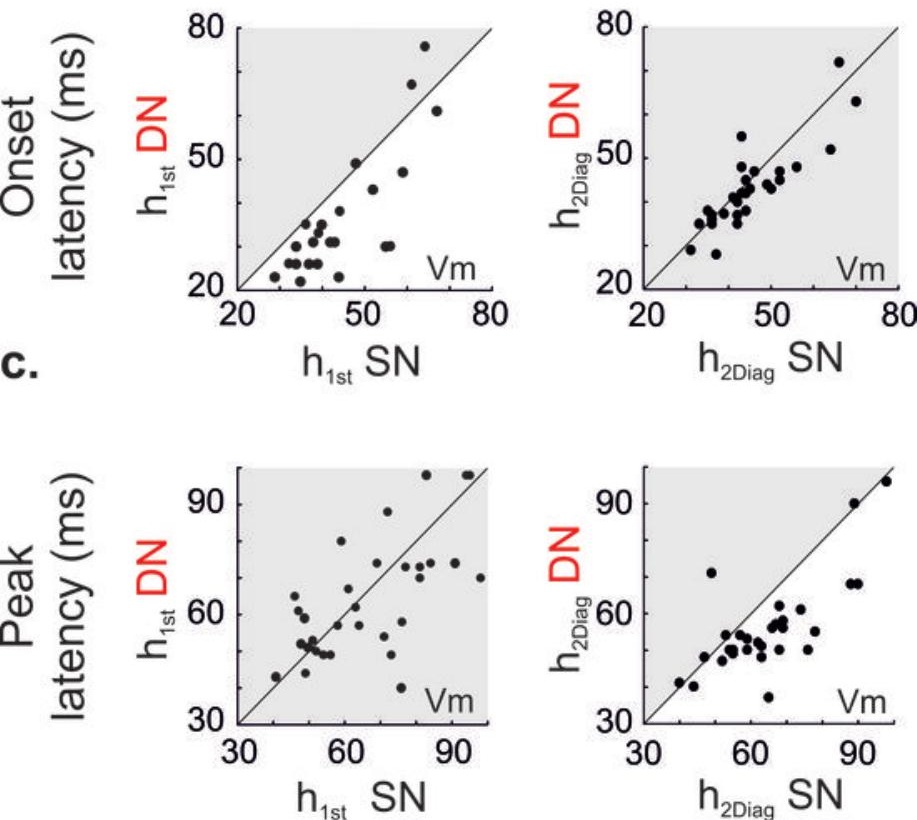

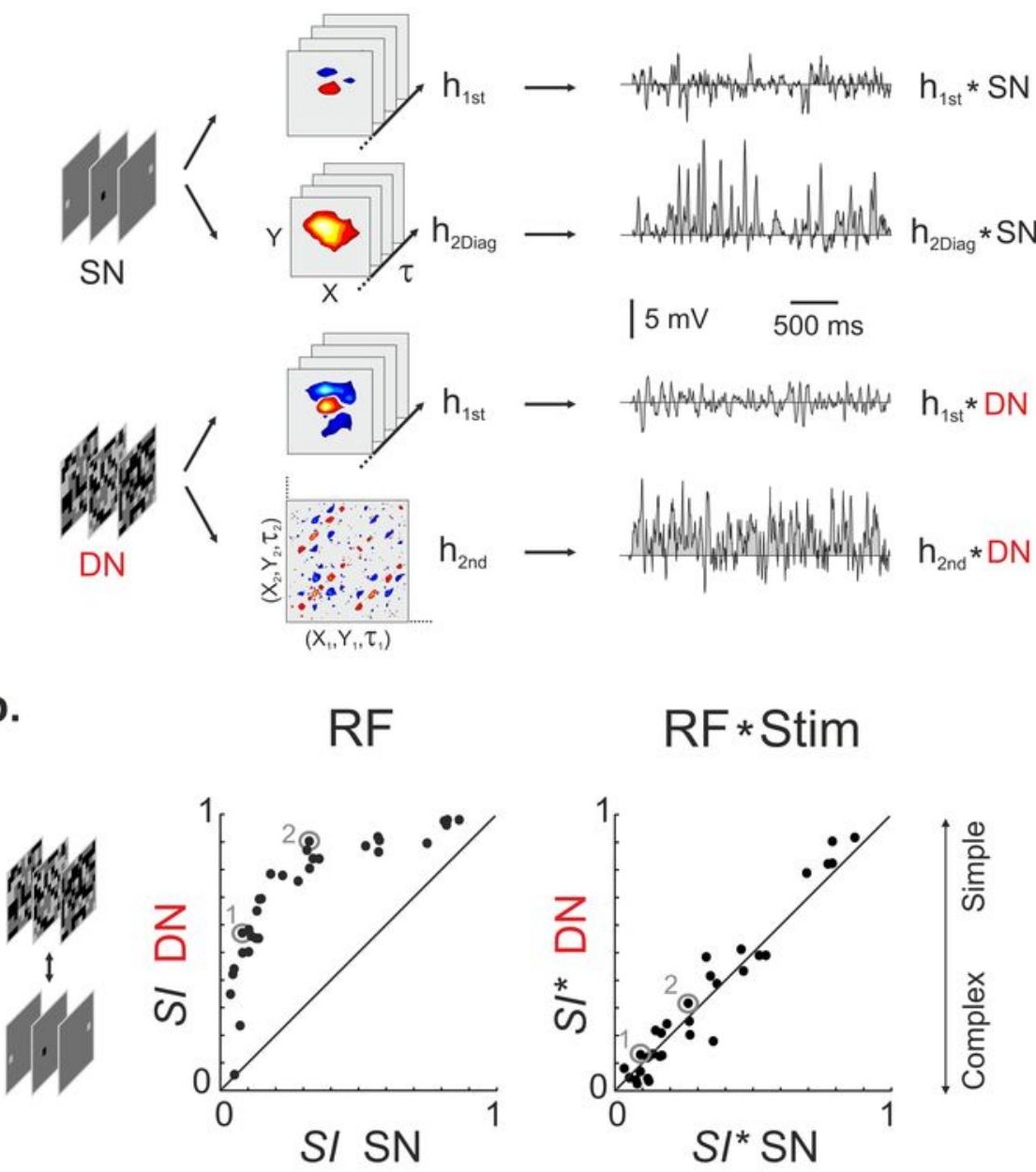

C.
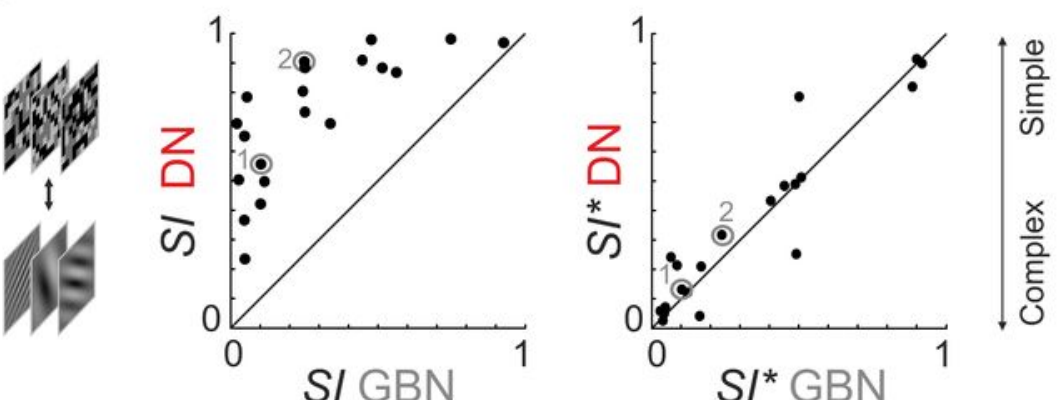
RF

$\mathrm{RF} *$ Stim

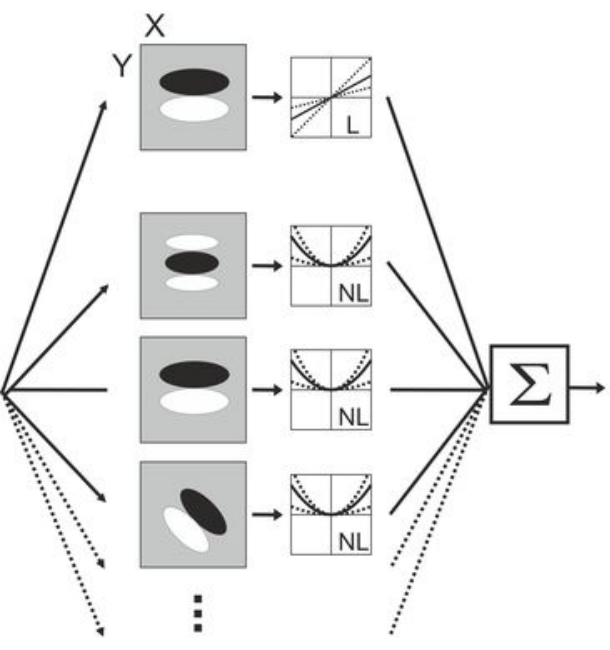

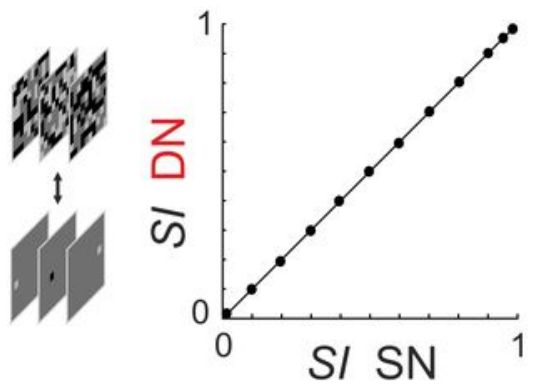

c.
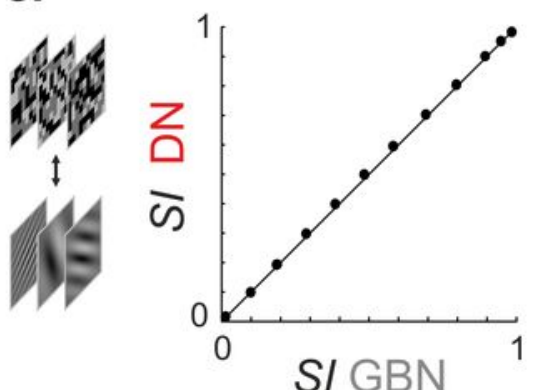
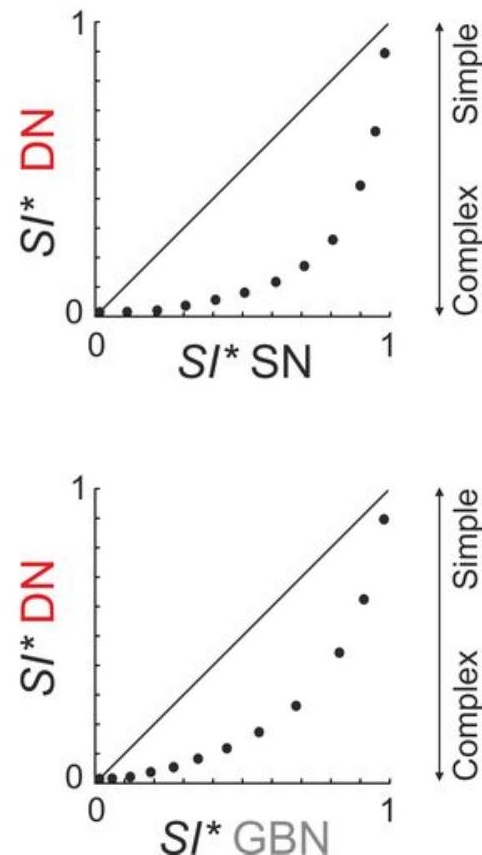
